\title{
User Content Delivery Service for Efficient POMA based LTE Channel Spectrum Scheduling Algorithm
}

\author{
V. Madhu Kumar, T. V. Ramana, Rajidi Sahithi
}

\begin{abstract}
Currently Long-Term Evolution (LTE) technologies are developing with an advanced network content delivery infrastructure sharing capabilities for different users and operators. Therefore, an efficient open-infrastructure of Position-orthogonal multiple access (POMA) based LTE networks with the content delivery capability is crucial to design and implement at this stage of research in LTE area. In this paper, the main contribution is the design and implementation of such LTE networks users open-infrastructure to enable the content delivery and sharing capabilities to be performed in virtual iterative precoding architectures. The proposed user content delivery infrastructure enables this service and sharing to the components of LTE architecture. A channel spectrum scheduling algorithm has been implemented through validation with test level experiments conducted to estimate the compatibility of LTE infrastructure virtualization in proposed open-infrastructure. The impact and scope of the proposed algorithm service can make the operational cost of the LTE mobile user to reduce.
\end{abstract}

Keywords : Long-term evolution (LTE), channel, service, sharing, scheduling

\section{INTRODUCTION}

The advancements in the infrastructure of LTE networks has made the research area in the current communication system through POMA [1][2], (PFSA) [3,4] and SDON [5]. Presently, the distribution of user content and its utilization through the LTE channel has the characteristics of antenna structures, user devices, nodes in denser urban and rural areas, different environment of user operating locations, distance communication links among the nodes, cost of communication links layed, and cost of equipment through the LTE infrastructure, resulting in difficult and different tasks available at the LTE channel infrastructure for user accurate communication system design.

Accordingly, the volume of user content has improved a-lot and it is a great challenge to provide faster user content transmission through communication system and also to be improved in mobility of user content in the LTE infrastructure network. The user content networking [6],[7] architecture is used to resolve the above said issues. Present communication system is made available with the presence of host-t-host communication, could be made advance for its future requirements which deals with the user content, its mobility for user in mobile at different state of services. The

Revised Manuscript Received on December 13, 2019.

* Correspondence Author

Mr. V. Madhu Kumar Assistant Professor, Dept. of ECE, JITS Warangal. madhuvjits@gmail.com

Dr. T. V. Ramana, Associate Professor, Dept. of ECE, GITAM (Deemed to be University),Visakhapatnam. teppala@gmail.com

Rajidi Sahithi, Assistant Professor, Dept. of ECE, CME College of Engineering and Technology, Hyderabad.sahithiramu@gmail.com usercontent delivery concentrates on providing the faster data rate with efficient flow of information with-in the secured links of communication path from the source of user content. The user content delivery service provides user-based routing, while masking the user device information, by operating through user secure messages for user content transmission through internet packet and user packet. Here, the user content through user packet is considered, in which the data related to user name, security information, and attributes of LTE communication networks are embedded through protocols.

In this paper, a User Content Delivery User Content Delivery Service (UCDS)-based efficient LTE channel spectrum scheduling algorithm is proposed. Through UCDS, a user content delivery mechanism is proposed to address the content and LTE infrastructure assessment in the LTE channel. The proposed delivery mechanism provides a user content handover prediction system to make the LTE mobile environment to guarantee the user data security.

The sections in this paper are arranged as, In second section the background for the content of the user services of LTE infrastructure is discussed. In third section proposed UCDS with LTE is discussed in detail. In fourth section the results were illustrated and finally, in section fifth the paper is concluded.

\section{BACKGROUND AND RELATED WORKS}

The frequency based spectrum sharing between the devices. LTE uses a space division method to separate between the user devices channel sharing. The spectrum sharing between the user devices made easy by managing the same MAC / Physical protocols. The transmission through the LTE devices is made enable by providing the simultaneous signal transmission. The spectrum sharing is among the cross technologies. WiFi and Zigbee spectrum sharing is made, which makes the power through the user device and network to handle more. To limit this, a similar sharing concept is applied in this paper to improve the bandwidth allocation by reducing the power levels.

Considering the POMA architecture [1] [2] based LTE infrastructure channel coverage and improving service capacity, a resource allocation process is defined and the network performance is through reducing the loss of information from the devices, and to improve LTE transmission quality a path dependent gateway is implemented[6].

All these lags in spectrum utilization as resources to allocate the user devices individually[7]. To limit this, a physical protocol is designed in this paper to share the PHY blocks between the

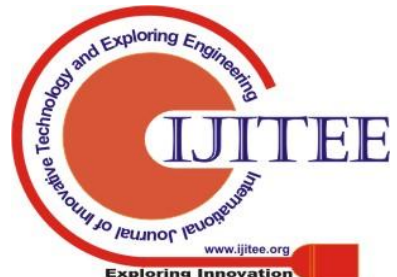


LTE devices and users. In all the above, there is some lags in content distribution and mobility operation due to inefficient network complexity. To limit this, a decentralized content storage is followed in the proposed UCDS to reduce the complexity and optimize the transmission efficiency.

\section{DESIGN AND IMPLEMENTATION OF PROPOSED WORK}

In this mobile LTE environment, the user is in mobile and moving away from the point of references. During the mobility the loss of content should not be caused due to lot of discontinuities in the communication links.

\section{A. Users Content Delivery Services (UCDS) delivery procedure:}

It is a seamless process of user content delivery without the loss of information in it because of the LTE infrastructure communication links. In the proposed work this is made possible through the UCDS LTE network. The LTE infrastructure components are illustrated in the figure 1, and with the functional flow the support of UCDS function and protocol are made in a procedure.

From the figure 1, the steps of procedure is shown below.

Step 1: UCDS procedure is enabled for User Equipment (UE) 1 .

Step 2: UE 1 request for connection establishment for the Content Source.

Step 3: Through eNodeB the UE 1 request is accepted and eNodeB sends the identification of UE 1 to the interface of SGW/PGW.

Step 4: Based on the Content Request, the content data packets are sent to eNodeB. The received content data is given to SGW/PGW, through the UCDS Content Delivery status.

Step 5: The received content data packets are sent to UE 1, if the matched content is acknowledged. And connection is terminated through the data packets received status.

Step 6: UCDS procedure is enabled for User Equipment (UE) 2.

Step 7: UE 2 request for connection establishment for the Content Source. Through eNodeB the UE 2 request is accepted and eNodeB sends the identification of UE 2 to the interface of SGW/PGW. Based on the Content Request, the content data packets are sent to eNodeB. The received content data is given to SGW/PGW, through the UCDS Content Delivery status.

Step 8: The received content data packets are sent to UE 2, if the matched content is acknowledged. And connection is terminated through the data packets received status.

\section{B. POMA based LTE Channel Spectrum Scheduling Algorithm:}

LTE infrastructure has a great design for its bandwidth and latency, but user content transmission system have a complex structure characteristics, as follows:

i) Due to limited data content resources, the user spectrum utilization and transmitter rate is reduced for user higher quantities of these.

ii) LTE network construction could not be applied to supportive technology because of its ratio of uplink and downlink channel available resources at the user end and by having high demand to downlink rather than uplink.

iii) The user content transmission is not accurate and fair, making the throughput of the LTE infrastructure system reduces.

iv) Lack of business requirement in the quality of LTE network services and poor uplink resource allocation, the power allocation of the existing LTE infrastructure is inaccurate.

Due to the above, the existing LTE infrastructure is not applicable to user content network resource scheduling services.

As shown in figure 2, the algorithm can be discussed with the following points.

1. At the first, the UCDS manages the service as user defined content scheduling and then prioritize the tasks according to user content data packets.

2. For the user defined content scheduling, the packet with higher priority having the shorter life cycle the user data packets have longer duration.

3. For the high prioritized tasks, the requirements are at an average level, making the bandwidth to reduce and user data packets retains more.

4. For the low prioritized tasks, the requirements are at low level, the throughput and fairness is increased and the transmission rate is increased.

\section{RESULTS AND DISCUSSION}

The simulation of the proposed work, the different user packets are considered and simulated data is recorded for the implementation execution. The analysis of the proposed work is dependent on the throughput of the user content, because the work is on scheduling the user content delivery service and compared with the proportional fair scheduling algorithm (PFSA) [3,4] and SDON [5] .

A. Throughput Metrics: The throughput simulation is compared with the PFSA, SDON and proposed work. The transmission of the business data with different priorities is considered here for comparison. The three priorities were considered here, by limiting the number of packets send by different services during the traffic simulation. Figure 3 shows the comparative results.

B. Delay Metrics: To verify the delay of content delivery the simulation time of the various user data packets were considered which are generated under different data request. The calculation of priority is made on the scheduling algorithm sorted in the proposed work. Figure 4 shows the comparative results. 


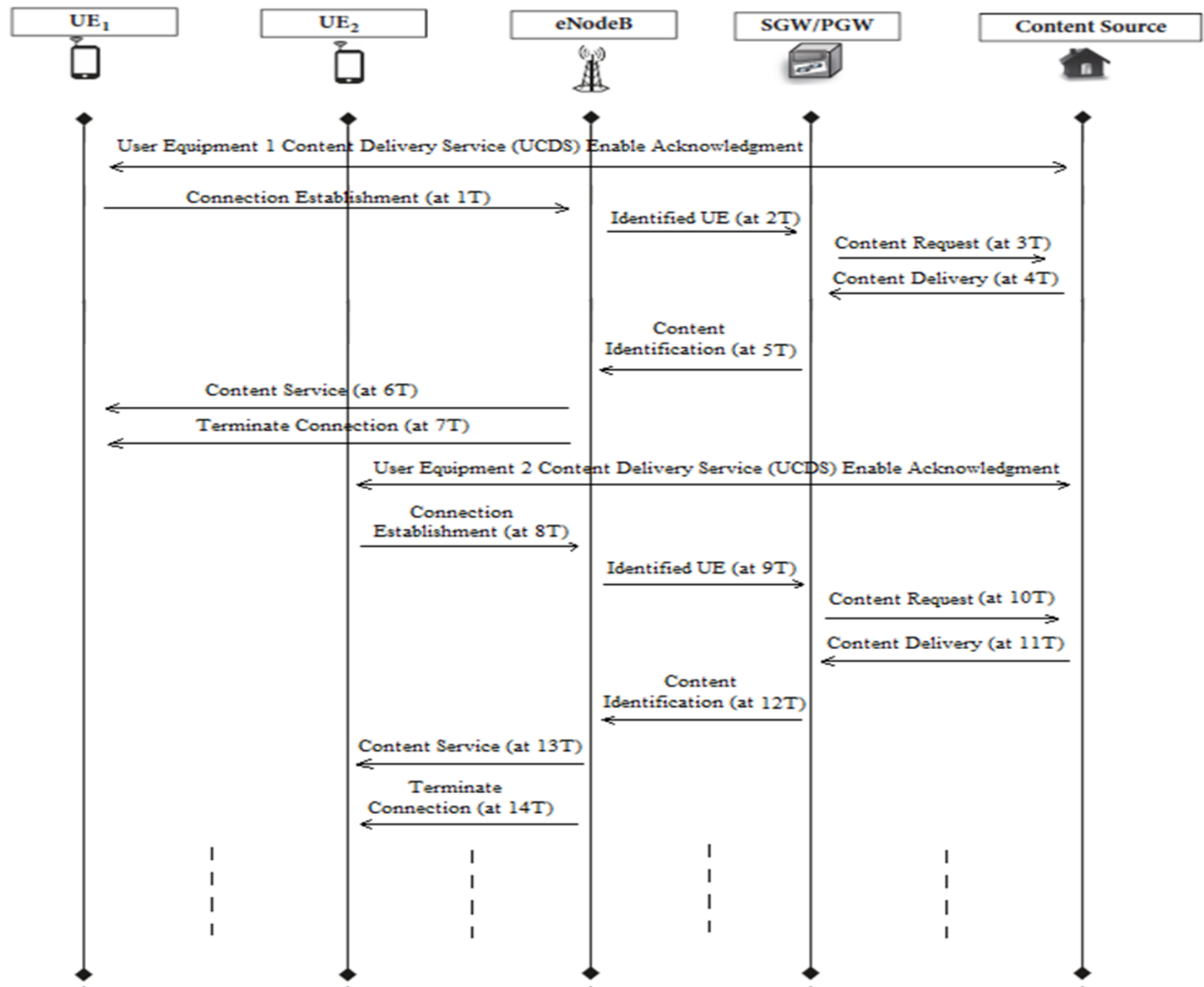

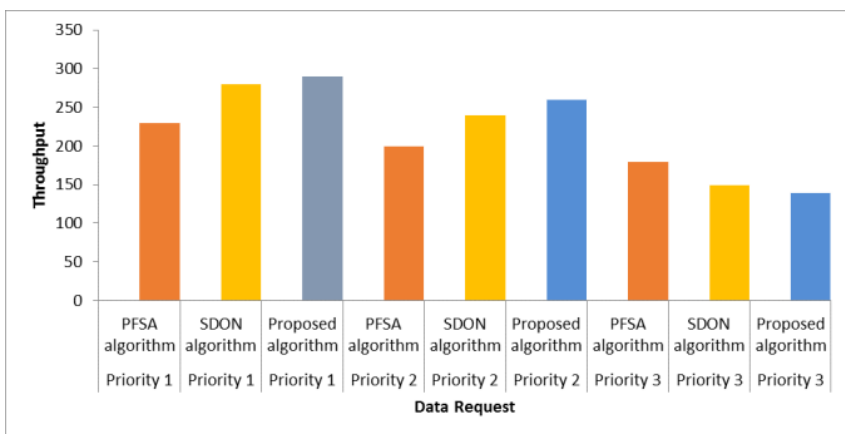

Fig: 3. The throughput of user content under different algorithm.

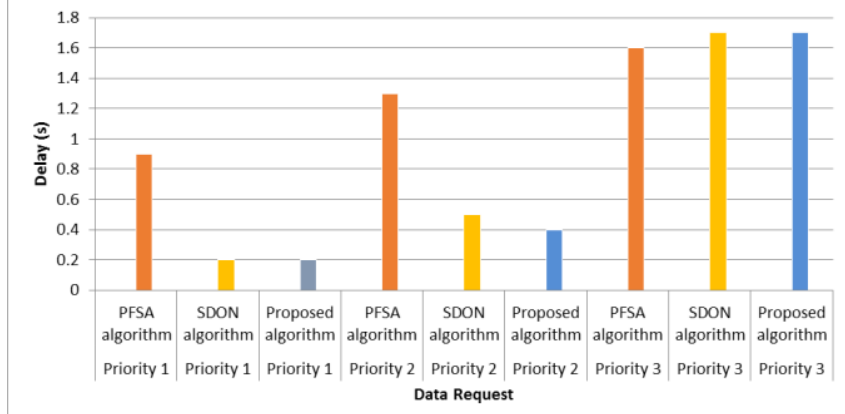

Fig: 4. The delay of user content under different algorithm. 


\section{CONCLUSIONS}

In this paper, a scheduling algorithm based on UCDS is proposed, which is an improved method for LTE infrastructure development, compared with the traditional approaches. Considering the throughput and delay constraints characteristics the quality of service is improved by the proposed work. Simulation results shows that the proposed scheduling algorithm is used to improve the LTE user content delivery through high throughput requirements. Under these constraints, the uplink resource allocation is possible by POMA method proposed in the previous works. The proposed work through POMA guarantee the better uplink mode of transmission for LTE communication link with the improved throughput and data packets delivery.

\section{REFERENCES}

1. V. M. Kumar and T. V. Ramana, "Position-based Fully-Scheduled Precoder Channel Strategy for POMA Structured LTE Network," 2019 IEEE International Conference on Electrical, Computer and Communication Technologies (ICECCT), Coimbatore, India, 2019, pp. 1-8.

2. V. M. Kumar and T. V. Ramana, "Virtual Iterative Precoding Based LTE POMA Channel Estimation Technique in Dynamic Fading Environments," 2019 International Journal of Innovative Technology and Exploring Engineering (IJITEE), ISSN: 2278-3075, Volume-8 Issue-6, April 2019, pp. 588-593.

3. C. Siqian, "Research on radio resource scheduling algorithm in LTE system," Harbin Institute of Technology, pp. 120-135, 2010.

4. L. Chen, J. Lu, and C. India, "LTE based on QoS business proportional fair scheduling algorithm," Optical Communication Research, vol. 5, pp. 64-67, 2012.

5. Ningzhe Xing, "Resources Scheduling Algorithm in Power Wireless Private Network Based on SDON", Hindawi, International Journal of Optics, Volume 2017, Article ID 2064638, 8 pages.

6. P. Y. Chang, J. M. Liang, J. J. Chen, K. R. Wu, and Y. C. Tseng, "Spatial and temporal aggregation for small and massive transmissions in LTE-M networks," in Proceedings of the IEEE Wireless Communications and Networking Conference, WCNC, San Francisco, CA, USA, March 2017.

7. D. Zhang, G. Li, K. Zheng, X. Ming, and Z. H. Pan, "An energy-balanced routing method based on forward-aware factor for wireless sensor networks," IEEE Transactions on Industrial Informatics, vol. 10, no. 1, pp. 766-773, 2014.

\section{AUTHOR PROFILE}

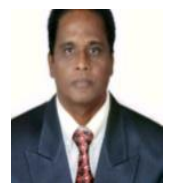

T. Venkata Ramana, as a diligent scholar has excelled in studies from his child hood. His dare to dream, to work smart and strive for nothing less than excellence and to enjoy the journey every step of the way made him to scale greater heights. He has obtained his Bachelor's Degree in Electronics \& Communications Engineering from Nagarjuna University. He has completed his Masters in Systems and Control Engineering from Osmania University. He has completed his Doctoral program Ph.D. in the area Satellite Communications from the Andhra University. He took up academic pursuit and served in an array of designations like Associate professor \& Coordinator for M. Tech (Evening programs) of GITAM University. He also involved himself in teaching and research tasks. Above all he took up a mission to ignite the young minds so as to nurture the students with the potential to reach greater horizons.

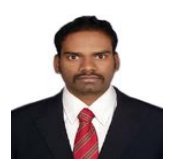

V.Madhu Kumar is a Research Scholar at GITAM University , Visakhapatnam and currently working as Assistant Professor in JITS (Autonomous), Narsampet, Waranga,Telangana. He has completed his Masters in DSCE from JNTU Hyderabad. He is the Member of IEEE and Life Member of ISTE. His research area are Wireless Communication and Signal Process-ing. His research topics are published in several National \& International Journals.

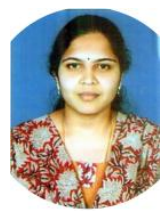

Rajidi Sahithi is a Research Scholar at GITAM University, Visakhapatnam and currently working as Assistant Professor in CMR College of Engineering and Technology,Hyderabad,Telangana.She has completed her Masters in VLSI System Design. Her research area are Wireless Communication and VLSI. Her research topics are published in several National \& International Journals. 